to choose the few particular members of the set of functions included in the calculation so that a good approximation is assured. A relatively simple calculation can be made to test the probable importance of omitted terms. Calculations on this principle have been carried out on a number of atoms and ions, including chlorine and sulphur, for which thirteen functions were used.

In the ensuing discussion, Dr. Boys (Cambridge) pointed out that the method used can be extended to find molecular wave-functions, the integral evaluation technique being largely applicable to this problem also. In reply to questions by Prof. Hartree and Dr. O. Bunemann (Cambridge), Dr. Boys said that the method is not likely to be of assistance in the nuclear field at present, since the accurate equations are not known with certainty. In answer to Dr. Buckingham, he said that electron affinities calculated by the method have so far shown disappointing lack of agreement with experiment.

After tea, two short contributions were given by visitors. Mr. W. A. Runciman (General Electric Co., Ltd., Wembley) described a problem connected with the fluorescence of a structure consisting of a potassium chloride lattice, in which some of the potassium ions are replaced by thallium. A first approximation to solution of this problem can be obtained by assuming the thallium ions to be in a free state, and an approximate evaluation of the wave functions by the self-consistent field method has been carricd out on the EDSAC.

The final contributor was Dr. R. H. Garstang (University College, London), who mentioned a problem he had met in the analysis of departures from Russell-Saunders coupling; this involved the determination of the diagonal elements of a matrix, given its non-diagonal elements and its characteristic values.

A. S. Douglas

\section{PHYSICAL FACTORS IN RELATION TO BIOLOGICAL ACTIVITY OF INSECTICIDES}

A MEETING of the Crop Protection Panel of the Agriculture Group of the Society of Chemical Industry was held in the rooms of the Chemical Society in London on February 16, when papers on "Physical Factors in Relation to Biological Activity of Insecticides" were read. Dr. R. A. E. Galley was in the chair.

Mr. C. T. Lewis (Imperial College of Science and Technology, London) discussed factors affecting the adhesion of dry particles of uniform size to active insect.s. Experiments with selected dyestuffs have shown that dry lipoid-soluble particles adhere more readily to the cuticle of blowflies, and to leaf wax, than do lipoid-insoluble particles possessing polar groups. Presumably the presence of polar groups lessens the attraction at the solid-solid interface due to Van der Waals forces. The phenomenon has an appreciable influence on the rate of accumulation of particles by flies exposed to deposits. Parallel experiments with insecticidal dusts have yet to be performed; but significant effects may reasonably be expected since contact insecticides are lipoid-soluble and most diluent dusts are calcium or magnesium silicates.
Mr. Lewis also considered the effect of the 'cleaning' reactions of a treated fly on the retention and location of accumulated particles. Particles are picked up from a surface on ventral tarsal spines and chemoreceptors, and transferred in cleaning movements to less exposed parts of the body. The frequent cleaning movements result in a balance between the weights of particles at different locations, the head at all times retaining about 7 per cent of the total, the legs 48 per cent, the wings 13 per cent and the abdomen 28 per cent. The pulvilli are not important sites of accumulation, retaining about 2 per cent, and experiments have shown that they are not preferred sites of entry of DDT.

The rate at which fine particles are discarded by a contaminated fly depends largely on the rate at which the continued cleaning movements deliver particles back to the tarsal spines, from which they are returned to the substrate. The rate of loss is rapid until the quantity retained by the fly falls to $20 \mu \mathrm{gm}$., but is very slow below $10 \mu \mathrm{gm}$.

Dr. A. H. MeIntosh (Rothamsted Experimental Station) dealt with the effect of certain conditions of test on the toxicity of a single insecticide, illustrated by reference to experiments in which insects were treated by dipping in aqueous suspensions of DDT. The weight retained depends upon the particle size, being greater for needle-shaped crystals than for colloidal particles. Temperature has a notable effect on toxicity, and the temperature coefficient, usually negative, varies with particle size and with humidity. High humidities accentuate the difference between the temperature coefficients of large and small particles.

The fact that poisons do not always have the same speed of action is an additional complication. The values obtained in relative toxicity tests may vary with the time at which observations of kill are made.

Dr. McIntosh also discussed the relation between the contact toxicity of insecticides and their fatsolubility. The first stage in the entry of a contact insecticide is assumed to be solution in the wax layer, and it is often implied that the higher the lipoidsolubility the more effective the poison is likely to be. An opposite conclusion can be deduced from Ferguson's principle of drug action, which indicates that different compounds have equal biological activity if applied at the same percentage saturation in the surrounding medium. Dr. McIntosh suggested that if this idea applied to the wax surrounding an insect, compounds of low fat-solubility should be the more effective. He pointed out, however, that there is no relation between the absolute toxicity of DDT and its analogues and lipoid-solubility. The view now held is that some degree of lipoid-solubility is necessary but it need not be high.

Experiments still in progress lead Dr. McIntosh to conclude that the difference in speed of action between colloid particles and crystals for a range of compounds of the DDT type may be related to the rate of solution of crystals in oil in vitro.

Dr. F. Barlow (Colonial Insecticide Research Unit, Porton) enumerated and discussed some factors of importance in the control of adult mosquitoes by residual spraying of houses. Mosquitoes are stimulated to flight after a short time in contact with insecticidal surfaces, and this fact increases the importance of physical factors affecting the availability of deposits.

The best formulation for deposits on absorbent walls is a wettable powder; the dispersing medium 
is absorbed, leaving insecticide particles freely exposed. On all types of surface, toxicity is inversely related to size of particle, because smaller particles are more easily picked up and retained by insects.

Dr. Barlow then dealt with factors affecting the persistence of deposits on various types of surface. Volatile insecticides exhibit decreasing residual action for a range of substrates from non-absorbent to absorbent. Increasing rates of evaporation and sorption into the substrate are responsible. Loss of insecticide has been found to be extremely rapid on certain adsorptive substrates and is due almost entirely to sorption; for example, on mud-blocks prepared from lateritic earths used for hut-building in several tropical countries.

Insecticides possessing fumigant action, for example, BHC and 'Aldrin', are effective for long periods after sorption, but others are ineffective after sorption, for example, DDT and 'Dieldrin'.

Finally, Dr. Barlow considered residual deposits of oil solutions. These deposits can be employed on non-absorbent surfaces, when formulations which do not crystallize are to be preferred because the recumbent crystals produced on such surfaces are not readily picked up by insects. By way of contrast, on fibrous absorbent surfaces, oil solutions are not available at first ; but after crystallization has occurred the crystals project from the superficial fibres and are readily detached by insects.

Opening the discussion, Dr. A. B. P. Page observed that all three speakers had dealt with ratedetermining processes. While there are big differences between fumigation and contact action, in each process accumulation and penetration of the toxicant are continuously occurring alongside toxification and detoxification within the insect. With fumigants, one measure of toxicity is the product of the concentration of the fumigant multiplied by the period of fumigation. In the same way the toxicity of a contact poison may be expected to be some integral function of the net amount of toxicant on the insect and the period of contact.

In certain fumigations a better measure of toxicity appears to be the amount of fumigant sorbed by the insects, and this relation implies dependence of toxicity on the shape of the concentration-time curve as well as on the area under it. An analogous relation may be found for contact poisons where large amounts of insecticide accumulated initially by the insect may greatly influence toxicity despite their rapid. removal by cleaning movements.

Dr. Page remarked that Dr. McIntosh had concerned himself with the solubility of poisons in the epicuticular wax layer, but had not mentioned the possibility of preferred sites of diffusion through the cuticle and access to specific sites of action. Such considerations are important. Referring to Dr. Barlow's account of the fumigant action of certain solid insecticides absorbed into porous substrates, he recalled an analogous case of absorption in bagged stored products treated with an insecticidal dust; wheat, for example, can absorb enough DD'T in the seed coats to enable the insecticide to act as a contact and stomach poison, whereas with $\mathrm{BHC}$ the fumigant action is all-important.

In reply to a question from Dr. J. R. Booer, Dr. McIntosh said that his colloidal suspensions were prepared in a standard way but he had not measured the size of particle.

Dr. J. C. McGowan pointed out that Ferguson's theory cannot be related to the system described by
Dr. McIntosh. If insects are treated with aqueous solutions of insecticide, then the theory can be used; but the solubility of DDT in water is so low that a lethal dose cannot be applied by such a method. Mr. G. F. H. Whitney observed that Ferguson's theory applies to substances showing 'physical' toxicity; a substance showing a toxicity greater than that expected by the application of Ferguson's theory invokes 'chemical' toxicity. Dr. McIntosh commented that he had not stated that Ferguson's theory is valueless, but that it is not useful in the case of DDT and its analogues. In answer to Mr. P. S. Hewlett, he agreed that he considers the action of DDT to be physical.

Mr. F. H. Rae asked whether Dr. Barlow advocated whitewashing huts before spraying, which he considered would not be a practicable procedure. Was sodium silicate useful and could Dr. Barlow suggest any other substance which might reduce losses due to sorption? Dr. Barlow said that the ideal would be to incorporate a substance in the spray which would block the capillary spaces in the substrate and thus reduce sorption. Size is not suitable for tropical use. The problem is being studied; but he had no practical recommendations to make at present.

Mr. P. Bracey asked if it is known whether BHC in the vapour phase enters an insect through the integument or through the spiracles; Dr. Page thought that no information is available.

Closing the discussion, Mr. W. H. Read stressed the practical importance of work in this field.

\section{THE PLACE OF UNIVERSITY GRADUATES IN INDUSTRY}

$\mathrm{T}$ WO most interesting points are featured in the report of the Universities and Industry Conference, organized by the Federation of British Industries and by the Committee of Vice-Chancellors and Principals of the Universities of the United Kingdom, and held at Ashorne Hill daring October $24-26,1952^{*}$ : the first is the further support which is forthcoming for the argument advanced in the last report from the Advisory Council for Scientific Policy that a new attitude towards industry and technology is desirable on the part of the schools and the universities; and the second is the large measure of agreement in addresses and discussions to the effect that, in consequence of the large proportion of talent which is now entering the universities, industry must necessarily rely to a much greater extent on recruitment from the universities to provide its future leaders (its chances of recruiting the requisite ability from those leaving school direct has been greatly diminished). The published report contains the addresses by the following : Sir John Maud on the use of the university graduate in the Civil Service and the qualities of the graduate which have proved most useful ; Sir Ewart Smith on the qualities industry requires in the university graduate and his role in industry; Dr. Eric Ashby on the ability of the universities, consistently with their other obligations, to turn out men with the qualities required by industry; and

* Report of the Universities and Industry Conference, organized by the Federation of British Industries and the Committee of ViceChancellors and Principals of the Universities of the United Kingdom, and held at Ashorne Hill, Leamington Spa, 24-26 October, 1952. Pp. 87. (London: F.B.I., 1953.) 58. 